\title{
PRIME AND SEARCH COMPUTABILITY, CHARACTERIZED AS DEFINABILITY IN CERTAIN SUBLANGUAGES OF CONSTRUCTIBLE $L_{\omega_{1}, \omega}$
}

BY

CARL E. GORDON

\begin{abstract}
The prime computable (respectively, search computable) relations of an arbitrary mathematical structure are shown to be those relations $R$ such that both $R$ and its complement are definable by disjunctions of recursively enumerable sets of quantifier free (respectively, existential) formulas of the first order language for the structure. The prime and search computable functions are also characterized in terms of recursive sequences of terms and formulas of this language.
\end{abstract}

1. Preliminaries. Let $\mathscr{U}=\left\langle A, R_{1}, \ldots, R_{a}, f_{1}, \ldots, f_{b}\right\rangle$ be a structure with each $R_{i}$ an $a_{i}$ place relation on $A$ and each $f_{i}$ a $b_{i}$ place function from $A$ to $A$. Let 0 be an object not in $A$, let $A^{0}=A \cup\{0\}$ and let $A^{*}$ be the closure of $A^{0}$ under ordered pair formation. For each $i=1, \ldots, a$ define $g_{i}^{*}$ on $A^{*}$ by:

$$
g_{i}^{*}\left(u_{1}, \cdots, u_{a_{i}}\right)= \begin{cases}0 & \text { if }\left\{u_{1}, \cdots, u_{a_{i}}\right\} \subset A \text { and } R_{i}\left(u_{1}, \cdots, u_{a_{i}}\right), \\ (0,0) & \text { otherwise. }\end{cases}
$$

For each $i=1, \ldots, b$ define $f_{i}^{*}$ on $A^{*}$ by

$$
f_{i}^{*}\left(u_{1}, \cdots, u_{b_{i}}\right)= \begin{cases}f_{i}\left(u_{1}, \cdots, u_{b_{i}}\right) & \text { if }\left\{u_{1}, \cdots, u_{b_{i}}\right\} \subset A, \\ (0,0) & \text { otherwise. }\end{cases}
$$

The extension of $A$ to $A^{*}$ is essential to the definitions of the classes of prime and search computable functions (cf. [3]). As in [3], we let $\pi$ and $\delta$ be respectively the left and right predecessor functions, corresponding to the ordered pair function $\lambda x y(x, y)$. The natural numbers are identified with elements of $A^{*}$ via the correspondence: $0=0, n+1=(n, 0)$. The set of natural numbers will be

Received by the editors June 4, 1973.

AMS (MOS) subject classifications (1970). Primary 02F 27; Secondary 02F 27.

Key words and phrases. Primitive computable, prime computable, search computable, infinitary language. 
denoted by $N$. Note that $N \cap A=\varnothing$. Given any set $C$, let $C^{(k)}$ denote the cartesian product of $C$ with itself $k$ times. We will be particularly interested in partial functions from sets of the form $N^{(p)} \times A^{(q)}$ into $N$ or into $A$. Such functions which are restrictions to $N^{(p)} \times A^{(q)}$ of functions on $A^{*}$ which are absolutely prime or search computable in $g_{1}^{*}, \ldots, g_{a}^{*}, f_{1}^{*}, \ldots, f_{b}^{*}$ will be called respectively U-prime-computable or $\mathrm{V}$-search-computable. The domain of an $\mathrm{U}$-prime-computable or $\mathcal{U}$-search-computable function will be called respectively a semi-2t-prime-comput able or semi-2t-search-computable relation. Among other results, it will be shown that a relation on $A$ is semi-2U-prime-computable if and only if it is definable by an infinite disjunction of a recursively enumerable set of quantifier free, finite formulas of the language of $\mathcal{U}$. It will also be shown that a relation on $A$ is semi-थ-search-computable if and only if it is definable by an infinite disjunction of a recursively enumerable set of existential, finite formulas of the language of $\mathscr{U}$.

2. The languages $Q F$ and $Q F^{*}$. Let $\mathfrak{Q}^{*}$ be the structure

$$
\left\langle A^{*}, A^{0}, \lambda x y(x, y), \pi, \delta, 0, R_{1}, \cdots, R_{a}, f_{1}^{*}, \cdots, f_{b}^{*}\right\rangle .
$$

$Q F$ and $Q F^{*}$ will be the quantifier free, finite languages for $\mathfrak{Q}$ and $\mathfrak{U}^{*}$ respectively. We will not distinguish between the elements of these languages and their "gödel numbers". Given natural numbers $n_{0}, \ldots, n_{k-1}$, let $\left\langle n_{0}, \ldots, n_{k-1}\right\rangle$ denote the product $\Pi_{i<k} P_{i}^{n_{i}}$, where $P_{0}=2$ and, for $i>0, P_{i}$ is the $i$ th odd prime. If $x=\left\langle n_{0}, \cdots, n_{k-1}\right\rangle$ then $x$ will be called a sequence number of length $k$ and we write $\operatorname{lh}(x)=k$ and $(x)_{i}=n_{i}(i=0, \ldots, k-1)$. In case $k=0, x=1$. If $y \in N$ is not a sequence number of length greater than $i$, let $(y)_{i}=0$.

$Q F$ is defined as follows.

(1) Variables. For each natural number $m,\langle 0, m\rangle$ is a variable (denoted by $\mathbf{V}_{m}$ ).

(2) Terms. The set of terms of $Q F$ is defined inductively by:

(i) Variables are terms.

(ii) If $1 \leq i \leq b$ and if $t_{1}, \cdots, t_{b_{i}}$ are terms then $\left\langle 1, i, t_{1}, \cdots, t_{b_{i}}\right\rangle$ is a term (denoted by $f_{i}\left(t_{1}, \cdots, t_{b_{i}}\right)$ ).

(3) Formulas. The set of formulas of $Q F$ is defined inductively by:

(i) $\langle 2\rangle$ is a formula (denoted by $\mathbf{T}$ and representing "true").

(ii) If $1 \leq i \leq a$ and if $t_{1}, \cdots, t_{a_{i}}$ are terms then $\left\langle 3, i, t_{1}, \cdots, t_{a_{i}}\right\rangle$ is a formula (denoted by $\mathbf{R}_{i}\left(t_{1}, \ldots, t_{a_{i}}\right)$ ).

(iii) If $\phi$ and $\psi$ are formulas then so are $\langle 4, \phi, \psi\rangle$ (denoted by $\phi \wedge \psi$ ), $\langle 5, \phi, \psi\rangle$ (denoted by $\phi \vee \psi$ ) and $\langle 6, \phi\rangle$ (denoted by $\urcorner \phi$ ).

$Q F$ is extended to $Q F^{*}$ as follows.

To the inductive definition of "term", add the clauses:

(iii) 0 is a term (denoted by 0 ).

(iv) If $s$ and $t$ are terms then so is $\langle 7, s, t\rangle$ (denoted by $\mathrm{p}(s, t)$ and representing the ordered pair of $s$ and $t$ ). 
(v) If $t$ is a term then so are $\langle 8, t\rangle$ and $\langle 9, t\rangle$ (denoted by $\pi t$ and $\delta t$ respectively).

To the inductive definition of "formula", add the clause:

(iv) If $t$ is a term then $\langle 10, t\rangle$ is a formula (denoted by $\mathrm{A}^{0}(t)$ and representing

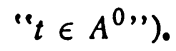

Satisfaction of formulas of $Q F$ in the structure $\mathcal{U}$ and of formulas of $Q F^{*}$ in the structure $\mathfrak{Q}^{*}$ is defined in the natural way, in light of the denotations used. In particular, the formula $T$ is true under all interpretations of variables. If $t$ is a term of $Q F$ (or $Q F^{*}$ ) with variables from $v_{1}, \ldots, v_{q}$ and if $x_{1}, \ldots, x_{q}$ is a sequence of elements of $A$ (or $A^{*}$ ) then $t\left[x_{1}, \ldots, x_{q}\right]^{q}$ will be used to denote the interpretation of $t$ determined by the interpretation of each $V_{i}$ as $x_{i}$. If $\phi$ is a formula of $Q F$ (or $Q F^{*}$ ) and if $x_{1}, \ldots, x_{q}$ is a sequence of elements of $A$ (or $A^{*}$ ), then $\phi\left[x_{1}, \ldots, x_{q}\right]$ will mean "the variables of $\phi$ are from $V_{1}, \ldots, v_{q}$ and $\phi$ is satisfied in $\mathcal{Q}$ (or $\hat{Q}^{*}$ ) by the interpretations of each $V_{i}$ as $x_{i}$ ".

Notice that if $t$ is a term and $\phi$ is a formula of $Q F$ and $x_{1}, \ldots, \dot{x}_{q}$ is a sequence of elements of $A$ then $t\left[x_{1}, \ldots, x_{q}\right]$ and $\phi\left[x_{1}, \ldots, x_{q}\right]$ have the same meanings with respect to $\mathfrak{I}^{*}$ as they have with respect to $\mathcal{U}$. If $\gamma$ is a term or formula of $Q F^{*}$ and $t_{1}, \ldots, t_{k}$ are terms of $Q F^{*}$, let $\gamma\left|t_{1}, \ldots, t_{k}\right|$ be the term or formula resulting from simultaneous substitution of $t_{1}, \ldots, t_{k}$ for all occurences of $\mathbf{V}_{1}, \ldots, \mathbf{V}_{k}$ respectively in $\gamma$. As a function of $\gamma, t_{1}, \ldots, t_{k} ; \gamma\left|t_{1}, \ldots, t_{k}\right|$ is the restriction of a primitive recursive function to a primitive recursive domain.

3. The main lemma. It will be shown (1) that prime and search computable relations and functions are definable by certain forms and (2) that relations and functions definable by those forms are prime or search computable. The latter (2) will probably be immediate to anyone conversant with the notions of prime and search computability. The former (1) is apparently somewhat surprising. The most difficult part of the proof is the proof of the main lemma (Lemma 1).

Lemma 1. For every $q$ place function $f$ on $A^{*}$ into $A^{*}$ wbich is absolutely primitive computable in $g_{1}^{*}, \ldots, g_{a}^{*}, f_{1}^{*}, \ldots, f_{b}^{*}$, there are total recursive functions $F$ and $G$ such that, for each $k \in N, F(k)$ and $G(k)$ are respectively a formula and a term of $Q F^{*}$ with variables from $\mathbf{V}_{1}, \ldots, \mathbf{V}_{q}$ and such that, for any $x_{1}, \ldots, x_{q} \in A^{*}$,

(i) there is a unique $k$ such that $F(k)\left[x_{1}, \ldots, x_{q}\right]$ and,

(ii) if $F(k)\left[x_{1}, \ldots, x_{q}\right]$ then $f\left(x_{1}, \ldots, x_{q}\right)=G(k)\left[x_{1}, \ldots, x_{q}\right]$.

The proof is by induction on the length of a primitive computable definition of $f_{0^{\circ}}^{\circ}$ The designations $\mathrm{CO}_{1}, \ldots, \mathrm{CO}_{a+b}, \mathrm{C} 2, \ldots, \mathrm{C} 7$ refer to clauses of the inductive definition of the class of primitive computable functions. If $f, G$ and $F$ are as above then we say that $(F, G)$ defermines $f$. In most cases we indicate a 
function $f$ and define functions $F$ and $G$, leaving it to the reader to verify that $F$ and $G$ are recursive and that $(F, G)$ determines $f$.

$$
\begin{gathered}
\mathrm{CO}_{i}(1 \leq i \leq a), f\left(t_{1}, \ldots, t_{a_{i}}, x_{1}, \ldots, x_{r}\right)=g_{i}^{*}\left(t_{1}, \ldots, t_{a_{i}}\right) . \\
F(0)=\mathbf{R}_{i}\left(\mathbf{V}_{1}, \cdots, \mathbf{V}_{a_{i}}\right), \quad F(1)=ר \mathbf{R}_{i}\left(\mathbf{V}_{1}, \ldots, \mathbf{V}_{a_{i}}\right), \quad F(k+2)=7 T . \\
G(0)=0, \quad G(k+1)=\mathbf{p}(0,0) .
\end{gathered}
$$$$
\mathrm{CO}_{a+i}(1 \leq i \leq b), f\left(t_{1}, \ldots, t_{b_{i}}, x_{1}, \ldots, x_{r}\right)=f_{i}^{*}\left(t_{1}, \ldots, t_{b_{i}}\right) .
$$$$
F(0)=\mathrm{T}, \quad F(k+1)=7 \mathrm{~T} . \quad G(k)=\mathrm{f}_{i}\left(\mathrm{~V}_{1}, \ldots, \mathrm{V}_{b_{i}}\right) .
$$

$\mathrm{C} 2, f\left(y, x_{1}, \ldots, x_{r}\right)=y$.

$$
F(0)=\mathbf{T}, \quad F(k+1)=7 \mathbf{T} . \quad G(k)=\mathbf{V}_{1} .
$$

C3, $f\left(s, t, x_{1}, \ldots, x_{r}\right)=(s, t)$.

$$
F(0)=\mathbf{T}, \quad F(k+1)=7 \mathbf{T} . \quad G(k)=\mathbf{p}\left(\mathbf{V}_{1}, \mathbf{V}_{2}\right) .
$$

$\mathrm{C} 4_{0}, f\left(y, x_{1}, \ldots, x_{r}\right)=\pi y$.

$$
F(0)=\mathbf{T}, \quad F(k+1)=7 \mathbf{T} . \quad G(k)=\pi \mathbf{V}_{1} .
$$

$C 4_{1}, f\left(y, x_{1}, \cdots, x_{r}\right)=\delta y$.

$$
F(0)=\mathbf{T}, \quad F(k+1)=7 \mathbf{T} . \quad G(k)=\delta \mathbf{V}_{1} \text {. }
$$

C5, $f\left(x_{1}, \ldots, x_{r}\right)=g\left(b\left(x_{1}, \ldots, x_{r}\right), x_{1}, \ldots, x_{r}\right)$. Assume, by the induction hypothesis, that there are functions $F_{1}, G_{1}, F_{2}$ and $G_{2}$ such that $\left(F_{1}, G_{1}\right)$ determines $g$ and $\left(F_{2}, G_{2}\right)$ determines $h$.

Letting $k_{i}=(k)_{i}$,

$$
\begin{aligned}
& F(k)=\left\{\begin{array}{l}
F_{2}\left(k_{0}\right) \wedge\left(F_{1}\left(k_{1}\right)\left|G_{2}\left(k_{0}\right), \mathbf{V}_{1}, \ldots, \mathbf{V}_{r}\right|\right) \text { if } k=\left\langle k_{0}, k_{1}\right\rangle, \\
\neg \mathbf{T} \text { if } k \text { is not a sequence number of length } 2 .
\end{array}\right. \\
& G(k)=G_{1}\left(k_{1}\right)\left|G_{2}\left(k_{0}\right), \mathbf{V}_{1}, \ldots, \mathbf{V}_{r}\right| \cdot
\end{aligned}
$$

C7, $f\left(x_{1}, \ldots, x_{r}\right)=g\left(x_{j+1}, x_{1}, \ldots, x_{j}, x_{j+2}, \ldots, x_{r}\right)$. C7 and C5 are handled similarly.

This completes all cases but C6 (C2 can be omitted when considering "absolute" computability).

$\mathrm{C} 6, f\left(y, x_{1}, \ldots, x_{r}\right)=g\left(y, x_{1}, \ldots, x_{r}\right)$ if $y \in A^{0}, f\left((s, t), x_{1}, \ldots, x_{r}\right)=$ $b\left(f\left(s, x_{1}, \cdots, x_{r}\right), f\left(t, x_{1}, \cdots, x_{r}\right), s, t, x_{1}, \cdots, x_{r}\right)$. Assume, by the induction hypothesis, that there are recursive functions $F_{1}, G_{1}, F_{2}$ and $G_{2}$ such that 
$\left(F_{1}, G_{2}\right)$ determines $g$ and $\left(F_{2}, G_{2}\right)$ determines $b$. Before proceeding with the definitions of $F$ and $G$, some development is required.

By a definition or proof by $A^{*}$-induction we will mean a definition or proof by induction with respect to the well-founded partial ordering on $A^{*}$ defined by:

(i) $x<(x, y)$ and $y<(x, y)$;

(ii) if $x<y$ and $y<z$ then $x<z$;

(iii) $x<y$ only as required by (i) and (ii).

This ordering will not be referred to again.

Given a sequence number $w=\left\langle x_{1}, \ldots, x_{j}\right\rangle$, let $w \hat{k}=\left\langle x_{1}, \ldots, x_{j}, k\right\rangle$ and $\widehat{k} w=\left\langle k, x_{1}, \ldots, x_{k}\right\rangle$. By a $0-1$-sequence is meant a sequence number $w$ such that $(w)_{i} \in\{0,1\}$ for all $i<\mathrm{lh}(w)$. By a bush is meant a finite, nonempty set $B$ of 0-1-sequences such that, for any $w$,

(i) $w^{\wedge} 0 \in B$ if and only if $w^{\wedge} 1 \in B$, and

(ii) if $w^{\wedge} 0 \in B$ then $w \in B$.

Notice that 1 is a member of every bush. An element $w$ of a bush $B$ will be called an endnode of $B$ if $w^{\wedge} 0 \notin B$. If $B$ is a bush, then a definition or proof $B$-induction is a definition or proof with respect to the well-founded (in fact finite) partial ordering < on $B$ defined by:

(i) $w^{\wedge} 0<w$ and $w^{\wedge} 1<w$ if $w^{\wedge} 0 \in B$;

(ii) if $x<y$ and $y<z$ then $x<z$;

(iii) $x<y$ only as required by (i) and (ii).

This ordering will not be referred to again. If $\beta$ is a function whose domain is a bush $B$ then $\beta$-induction will mean $B$-induction.

We associate with each $0-1$-sequence $w$, a term $t(w)$ of $Q F^{*}$. The definition of $t(w)$ is by induction on $\mathrm{lh}(w)$.

$$
t(1)=\mathbf{V}_{1}, \quad t\left(w^{\wedge} 0\right)=\pi t(w), \quad t\left(w^{\wedge} 1\right)=\delta t(w) .
$$

It is easy to show that, for each $0-1$-sequence $w$,

$$
t(\widehat{O w})=t(w)\left|\pi \mathrm{V}_{1}\right|, \quad t(\widehat{1})=t(w)\left|\delta \mathrm{V}_{1}\right| \cdot
$$

Given a function $\beta$ from a bush $B$ into $N$, we assign to each $w \in B$ a term $f(w)$ and a formula $\phi(w)$. The functions $\tau$ and $\phi$ are defined simultaneously by $\beta$-induction. Write ${ }^{\beta}{ }^{\beta}$ and $\phi^{\beta}$ to indicate the dependence upon $\beta$. Recall that $\left(F_{1}, G_{1}\right)$ determines $g$ and $\left(F_{2}, G_{2}\right)$ determines $b$. If $w$ is an endnode of $B$,

$$
\phi(w)=\mathbf{A}^{0}((w)) \wedge F_{1}(\beta(w))|t(w)| \text { and } \gamma(w)=G_{1}(\beta(w))|t(w)| .
$$

If $w$ is an element of, but not an endnode of $B$,

$$
\begin{aligned}
\phi(w)= & \left.\phi\left(w^{\wedge} 0\right) \wedge \phi\left(w^{\wedge} 1\right) \wedge\right\urcorner A^{0}(t(w)) \\
& \wedge F_{2}(\beta(w))\left|r\left(w^{\wedge} 0\right), r\left(w^{\wedge} 1\right), t\left(w^{\wedge} 0\right), t\left(w^{\wedge} 1\right), \mathbf{V}_{2}, \cdots, \mathbf{V}_{r+1}\right|
\end{aligned}
$$


and

$$
x(w)=G_{2}(\beta(w))\left|x\left(w^{\wedge} 0\right), x\left(w^{\wedge} 1\right), t\left(w^{\wedge} 0\right), t\left(w^{\wedge} 1\right), V_{2}, \cdots, V_{r+1}\right| \cdot
$$

Lemma 1.1. For $i=0,1,2$, let $\beta_{i}$ be a function from a bush $B_{i}$ into $N$. Let $\phi_{i}=\phi^{\beta_{i}}$ and $r_{i}=\tau^{\beta_{i}}$. Assume that $B_{2}=\{1\} \cup\left\{0\right.$ w: $\left.w \in B_{0}\right\} \cup\left\{1 w: w \in B_{1}\right\}$, $\beta_{2}(0 \widetilde{w})=\beta_{0}(w)$ and $\beta_{2}(\digamma w)=\beta_{1}(w)$. Then:

(a) for each $w \in B_{0}, \phi_{2}(0 \frown w)=\phi_{0}(w)\left|\pi V_{1}\right|$ and $\tau_{2}(0 \frown w)=\tau_{0}(w)\left|\pi V_{1}\right|$, and

(b) for each $w \in B_{1}, \phi_{2}(\mathcal{F} w)=\phi_{1}(w)\left|\delta V_{1}\right|$ and $\tau_{2}(1-w)=\tau_{1}(w)\left|\delta V_{1}\right|$.

Proof of (a) by $B_{0}$ induction. If $w$ is an endnode of $B_{0}$, then $0 w$ is an endnode of $B_{2}$ and $\phi_{0}(w)=\mathbf{A}^{0}(t(w)) \wedge F_{1}\left(\beta_{0}(w)\right)|t(w)|$. Therefore, $\phi_{0}(w)\left|\pi \mathbf{V}_{1}\right|=$ $\mathbf{A}^{0}\left(t(w)\left|\pi \mathbf{V}_{1}\right|\right) \wedge F_{1}\left(\beta_{0}(w)\right)|t(w)| \pi \mathbf{V}_{1} \|$. So, by (3.2),

$$
\phi_{0}(w)\left|\pi V_{1}\right|=\mathbf{A}^{0}(t(\widetilde{w} w)) \wedge F_{1}\left(\beta_{0}(w)\right)|t(\widetilde{0} w)| \text {. }
$$

Since $\beta_{0}(w)=\beta_{2}(0 \widetilde{w}), \phi_{0}(w)\left|\pi \mathbf{V}_{1}\right|=\phi_{2}(0 \widetilde{w})$. Similarly, $\tau_{0}(w)\left|\pi \mathbf{V}_{1}\right|=\tau_{2}(0 w w)$. If $w$ is an element of but not an endnode of $B_{0}$, then $w^{\wedge} 0$ and $w^{\wedge} 1$ are elements of $B_{0}$ and, by the $B_{0}$-induction hypothesis, $\phi_{0}\left(w^{\wedge} i\right)\left|\pi V_{1}\right|=\phi_{2}\left(0 \frown w^{\wedge} i\right)$ and $\tau_{0}\left(w^{\wedge} i\right)\left|\pi \mathbf{V}_{1}\right|=\tau_{2}\left(\sigma^{w} i\right)$, for $i=1,2$. By (3.2), $t\left(w^{\wedge} i\right)\left|\pi \mathbf{V}_{1}\right|=t\left(0^{\wedge}{ }^{\wedge} i\right)$ and $t(w)\left|\pi \mathbf{V}_{1}\right|=t\left(\sigma^{2} w\right)$. Herice

$$
\begin{aligned}
& \left.\phi_{0}(w)\left|\pi V_{1}\right|=\phi_{2}\left(\widetilde{0} \hat{w}^{\wedge} 0\right) \wedge \phi_{2}\left(\widetilde{w^{\wedge}} 1\right) \wedge\right\urcorner A^{0}(t(\widetilde{w}))
\end{aligned}
$$

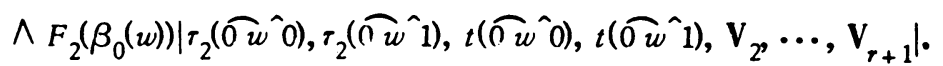

Since $\beta_{0}(w)=\beta_{2}(0 \widetilde{w}), \phi_{0}(w)\left|\pi \mathbf{V}_{1}\right|=\phi_{2}(0 \widetilde{w})$. Similarly, $\tau_{0}(w)\left|\pi \mathbf{V}_{1}\right|=\tau_{2}(0 \widetilde{w})$. This completes the proof of part (a). The proof of part (b) is similar. Now let $\kappa$ be any effective one-one enumeration of functions $\beta$ into $N$ whose domains are bushes and define functions $F$ and $G$ by: $F(k)=\phi^{K(k)}(1), G(k)=\tau^{K(k)}(1)$. By Church's thesis, $F$ and $G$ are recursive.

Lemma 1.2. Let $\mathrm{x}=x_{1}, \ldots, x_{r}$ be a sequence of elements of $A^{*}$. Then (a) for each $y \in A^{*}$ there is a unique number $k$ sucb that $F(k)[y, \mathbf{x}]$ and (b) if $F(k)[y, \mathbf{x}]$ then $G(k)[y, \mathbf{x}]=f(y, \mathbf{x})$.

Proof by $A^{*}$-induction on $y$. If $y \in A^{0}$ choose $k$ such that $\kappa(k)$ is that unique function $\beta$ with domain $\{1\}$ such that $F_{1}(\beta(1))[y, \mathbf{x}]$. Then $F(k)=$ $\phi^{\beta}(1)=\mathbf{A}^{0}\left(V_{1}\right) \wedge F_{1}(\beta(1))$ and $G(k)=\tau^{\beta}(1)=G_{1}(\beta(1))$. Therefore $F(k)[y, \mathbf{x}]$ and $G(k)[y, \mathbf{x}]=g(y, \mathbf{x})=f(y, \mathbf{x})$. Suppose $F\left(k^{\prime}\right)[y, \mathbf{x}]$. Let $\kappa\left(k^{\prime}\right)=\beta^{\prime}$ so $F\left(k^{\prime}\right)=$ $\phi^{\beta^{\prime}}(1)$. If 1 is not an endnode of $\beta^{\prime}$ then $\phi^{\beta^{\prime}}(1)[y, \mathbf{x}]$ implies that $7 \mathbf{A}^{0}\left(\mathbf{V}_{1}\right)[y, \mathbf{x}]$, which implies that $y \notin A^{0}$. Therefore 1 is an endnode of domain $\left(\beta^{\prime}\right)$,(i.e., domain $\left.\left(\beta^{\prime}\right)=\{1\}\right)$ and $\phi^{\beta^{\prime}}(1)[y, \mathbf{x}]=F_{1}\left(\beta^{\prime}(1)\right)[y, \mathbf{x}]$. This uniquely determines $\beta^{\prime}(1)$ so $\beta=\beta^{\prime}$. Now assume $y=(s, t)$. Let $k_{0}$ and $k_{1}$ be the unique numbers such that $F\left(k_{0}\right)[s, \mathbf{x}]$ and $F\left(k_{1}\right)[t, \mathbf{x}]$ and assume, by the induction 
hypothesis that $G\left(k_{0}\right)[s, \mathbf{x}]=f(s, \mathbf{x})=u$ and $G\left(k_{1}\right)[t, \mathbf{x}]=f(t, \mathbf{x})=v$. Let $j$ be that unique number such that $F_{2}(j)[u, v, s, t, x]$. Let $\kappa\left(k_{0}\right)=\beta_{0}, \kappa\left(k_{1}\right)=\beta_{1}$ and let $\beta_{2}$ be that function such that $\beta_{2}(1)=j$ and $\beta_{0}, \beta_{1}$ and $\beta_{2}$ are related as in Lemma 1.1. Choose $k$ such that $\kappa(k)=\beta_{2}$. Letting $\phi_{i}=\phi^{\beta_{i}}$ and $\tau_{i}=\tau^{\beta_{i}}$ $(i=0,1,2)$, we have

$$
\begin{aligned}
F(k)= & \phi_{2}(1)=\phi_{2}(\langle 0\rangle) \wedge \phi_{2}(\langle 1\rangle) \wedge \neg \mathrm{A}^{0}\left(\mathrm{~V}_{1}\right) \\
& \wedge F_{2}(j)\left|\tau_{2}(\langle 0\rangle), \tau_{2}(\langle 1\rangle), \pi \mathrm{V}_{1}, \delta \mathrm{V}_{1}, \mathrm{~V}_{2}, \ldots, \mathrm{V}_{r+1}\right| \\
= & \left.\dot{\phi}_{0}(1)\left|\pi \mathrm{V}_{1}\right| \wedge \phi_{1}(1)\left|\delta \mathrm{V}_{1}\right| \wedge\right\urcorner \mathrm{A}^{0}\left(\mathrm{~V}_{1}\right) \\
& \wedge F_{2}(j)\left|\tau_{0}(1)\right| \pi \mathrm{V}_{1}\left|, \tau_{1}(1)\right| \delta \mathrm{V}_{1}\left|, \pi \mathrm{V}_{1}, \delta \mathrm{V}_{1}, \mathrm{~V}_{2}, \ldots, \mathrm{V}_{r+1}\right|
\end{aligned}
$$

Similarly $G(k)=G_{2}(j)\left|\tau_{0}(1)\right| \pi \mathrm{V}_{1}\left|, r_{1}(1)\right| \delta \mathrm{V}_{1}\left|, \pi \mathrm{V}_{1}, \delta \mathrm{V}_{1}, \mathrm{~V}_{2}, \ldots, \mathrm{V}_{r+1}\right|$. Now it is easy to verify that

(a) $F(k)[y, \mathbf{x}]$ if and only if $F\left(k_{0}\right)[s, \mathbf{x}], F\left(k_{1}\right)[t, \mathbf{x}], y \notin A^{0}$ and $F_{2}(j)[u, v, s, t, \mathbf{x}]$, and that

(b) $G(k)[y, \mathbf{x}]=G_{2}(j)[u, v, s, t, \mathbf{x}]=b(u, v, s, t, \mathbf{x})=/(y, \mathbf{x})$.

To show the uniqueness of such $k$, assume that $F\left(k^{\prime}\right)[y, \mathbf{x}]$. Let $k\left(k^{\prime}\right)=\beta^{\prime}$. If 1 is an endnode of domain $\left(\beta^{\prime}\right)$, then $F\left(k^{\prime}\right)\left[y^{\prime}, \mathbf{x}\right]$ implies that $\phi^{\beta^{\prime}}(1)[y, \mathbf{x}]$ which implies that $y \in A^{0}$. Hence 1 is not an endnode. Let $\beta_{0}^{\prime}(w)=\beta^{\prime}(0 \widetilde{w})$ and $\beta_{1}^{\prime}(w)=\beta^{\prime}(1 \widetilde{w})$, for all $w$ in the appropriate domains. Then $\beta_{0}^{\prime}, \beta_{1}^{\prime}$ and $\beta^{\prime}$ are related as in Lemma 1.1. Choose $k_{0}^{\prime}$ and $k_{1}^{\prime}$ such that $\kappa\left(k_{0}^{\prime}\right)=\beta_{0}^{\prime}$ and $\kappa\left(k_{1}^{\prime}\right)=\beta_{1}^{\prime}$. From the assumption that $F\left(k^{\prime}\right)[y, \mathbf{x}]$, it can be concluded that $F\left(k_{j}^{\prime}\right)[s, \mathbf{x}]$, $F\left(k_{1}^{\prime}\right)[t, \mathbf{x}]$ and $F_{2}\left(\beta^{\prime}(1)\right)[u, v, s, t, \mathbf{x}]$ and hence that $k_{0}^{\prime}=k_{0}, k_{1}^{\prime}=k_{1}$ and $\beta^{\prime}(1)=\beta(1)$. Therefore $\beta=\beta^{\prime}$. This completes the proof of Lemma 1.2 and hence the proof of Lemma 1 .

4. Embedding $Q F$ in $Q F^{*}$. Let $T m^{*}$ be the smallest set of terms of $Q F^{*}$ containing all the terms of $Q F$, the term 0 and containing $p(s, t)$ whenever it contains $s$ and $t$. $T m^{*}$ contains a numeral $\mathrm{m}$ for each natural number $m$, i.e., $\mathbf{0}=0, \mathrm{~m}+\mathbf{l}=\mathbf{p}(\mathrm{m}, \mathbf{0})$.

Lemma 2. There is a total recursive function $\eta$ such that if $t$ is a term and $\phi$ is a formula of $Q F^{*}$ with variables from $\left\{V_{1}, \ldots, V_{k}\right\}$ and if $x_{1}, \ldots, x_{k}$ is a sequence of elements of $A$ then

(i) $\eta(t)$ is a term of $\mathrm{Tm}^{*}$ with variables from $\left\{\mathbf{V}_{1}, \ldots, \mathbf{V}_{k}\right\}$,

(ii) $\eta(t) \in Q F$ if and only if $t\left[x_{1}, \ldots, x_{k}\right] \in A$,

(iii) for $m \in N, \eta(t)=\mathbf{m}$ if and only if $t\left[x_{1}, \ldots, x_{k}\right]=m$,

(iv) $t\left[x_{1}, \ldots, x_{k}\right]=\eta(t)\left[x_{1}, \ldots, x_{k}\right]$,

(v) $\eta(\phi)$ is a formula of $Q F$ with variables from $\left\{\mathbf{V}_{1}, \ldots, \mathbf{V}_{k}\right\}$ and

(vi) $\phi\left[x_{1}, \ldots, x_{k}\right]$ if and only if $\eta(\phi)\left[x_{1}, \ldots, x_{k}\right]$. 
Proof. If $z$ is neither a term nor a formula of $Q F^{*}$, let $\eta(z)=0$. Define $\eta$ on the terms of $Q F^{*}$ by recursion and the following cases:

$$
\mathrm{T}_{1} \cdot \eta(0)=0 .
$$

$\mathrm{T}_{2} \cdot \eta\left(\mathbf{V}_{i}\right)=\mathbf{V}_{i}$, for each variable $\mathbf{V}_{i}$.

$\mathrm{T}_{3} . \quad \eta(\pi t)= \begin{cases}0 & \text { if } \eta(t)=0, \\ 1 & \text { if } \eta(t) \in Q F, \\ u & \text { if } \eta(t)=\mathrm{p}(u, v), \\ 0 & \text { otherwise. (This case never occurs.) }\end{cases}$

$\mathrm{T}_{4^{\circ}} \eta(\delta t)= \begin{cases}0 & \text { if } \eta(t)=0, \\ 1 & \text { if } \eta(t) \in Q f, \\ v & \text { if } \eta(t)=\mathbf{p}(u, v), \\ 0 & \text { otherwise. (This case never occurs.) }\end{cases}$

$\mathrm{T}_{5} \cdot \eta(\mathrm{p}(s, t))=\mathbf{p}(\eta(s), \eta(t))$.

$\mathrm{T}_{6^{*}} \quad \eta\left(\mathrm{f}_{i}\left(t_{1}, \cdots, t_{b_{i}}\right)\right)= \begin{cases}\mathrm{f}_{i}\left(\eta\left(t_{1}\right), \cdots, \eta\left(t_{b_{i}}\right)\right) & \text { if } \eta\left(t_{1}\right), \cdots, \eta\left(t_{b_{i}}\right) \in Q F, \\ 1 & \text { otherwise. }\end{cases}$

Define $\eta$ on the formulas of $Q F^{*}$ by recursion and the following cases:

$$
\begin{aligned}
& \mathbf{F}_{1} \cdot \eta\left(\mathbf{A}^{0}(t)\right)= \begin{cases}\mathbf{T} & \text { if } \eta(t)=0 \text { or } \eta(t) \in Q F, \\
7 \mathbf{T} & \text { otherwise. }\end{cases} \\
& \mathrm{F}_{2^{*}} \eta\left(\mathbf{R}_{i}\left(t_{1}, \cdots, t_{a_{i}}\right)\right)= \begin{cases}\mathbf{R}_{i}\left(\eta\left(t_{1}\right), \cdots, \eta\left(t_{a_{i}}\right)\right) & \text { if } \eta\left(t_{1}\right), \cdots, \eta\left(t_{a_{i}}\right) \in Q F, \\
7 \mathrm{~T} & \text { otherwise. }\end{cases} \\
& \mathrm{F}_{3^{\circ}} \quad \eta(\mathrm{T})=\mathrm{T} \\
& \left.\left.\mathrm{F}_{4^{\circ}} \quad \eta(\urcorner \phi\right)=\right\urcorner \eta(\phi), \eta(\phi \wedge \psi)=\eta(\phi) \wedge \eta(\psi) \text { and } \eta(\phi \vee \psi)=\eta(\phi) \vee \eta(\psi) \text {. }
\end{aligned}
$$

That $\eta$ is recursive follows from the recursive definitions of $Q F$ and $Q F^{*}$. Parts (i) and ( $v$ ) of Lemma 2 are immediate by induction over the definitions of term and formula respectively.

Lemma 2.1. If $t$ is a term of $\mathrm{Tm}^{*}, x_{1}, \ldots, x_{k}$ is a sequence of elements of $A$ and $t\left(x_{1}, \ldots, x_{k}\right) \in A$ then $t \in Q F$. 
Proof. If $t \in T m^{*}$ and $t \notin Q F$ then either $t=0$ or $t$ is of the form $\mathrm{p}(u, v)$. In either case $t\left[x_{1}, \ldots, x_{k}\right] \in A$.

Lemma 2.2. If $t$ is a term of $\mathrm{Tm}^{*}, x_{1}, \ldots, x_{k}$ is a sequence of elements of $A, m \in N$ and $t\left[x_{1}, \cdots, x_{k}\right]=m$, then $t=\mathrm{m}$.

The proof is easy by induction over the inductive definition of $\mathrm{Tm}^{*}$.

Now part (iv) of Lemma 2 can be proved by induction over the definition of $\eta$. The proof is straightforward except that, in case $\mathrm{T}_{6}\left(t=\mathrm{f}_{i}\left(t_{1}, \cdots, t_{b_{i}}\right)\right)$, Lemma 2.1 is needed. Parts (ii) and (iii) follow immediately from parts (i) and (iv) and Lemmas 2.1 and 2.2. Part (vi) can be proved by induction over the definition of $\eta$. In cases $F_{1}$ and $F_{2}$, Lemmas 2.1 and 2.2 are needed.

5. Prime computable functions. Let $\mu$ be the minimalization operator.

Theorem 1(a). If $f$ is an $\mathscr{U}$-prime-computable function from $N^{(p)} \times A^{(q)}$ into $A$ then there are total recursive functions $F$ and $G$ from $N^{(p+1)}$ into the sets of formulas and terms respectively of $Q F$ such that, for any $\left(n_{1}, \ldots, n_{p}, x_{1}, \cdots, x_{q}\right)$ $\epsilon N^{(p)} \times A^{(q)}$

$$
\begin{aligned}
& f\left(n_{1}, \cdots, n_{p}, x_{1}, \cdots, x_{q}\right) \\
& \quad=G\left(\mu k F\left(k, n_{1}, \cdots, n_{p}\right)\left[x_{1}, \cdots, x_{q}\right], n_{1}, \cdots, n_{p}\right)\left[x_{1}, \cdots, x_{q}\right] .
\end{aligned}
$$

(b) If $f$ is an 2 -prime-computable function from $N^{(p)} \times A^{(q)}$ into $N$ then there is a total recursive function $F$ from $N^{(p+1)}$ into the set of formulas of $Q F$ and a total recursive function $H$ from $N^{(p+1)}$ into $N$ such that, for $\left(n_{1}, \ldots, n_{p}\right.$, $\left.x_{1}, \cdots, x_{q}\right) \in N^{(p)} \times A^{(q)}$,

$$
f\left(n_{1}, \cdots, n_{p}, x_{1}, \cdots, x_{q}\right)=H\left(\mu k F\left(k, n_{1}, \cdots, n_{p}\right)\left[x_{1}, \cdots, x_{q}\right], n_{1}, \cdots, n_{p}\right) .
$$

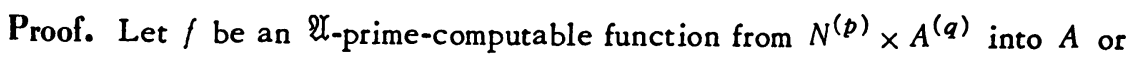
into $N$. By Remark 10 of [3], there is a function $U$ and a relation $T$, each absolutely primitive computable in $g_{1}^{*}, \ldots, g_{a}^{*}, f_{1}^{*}, \ldots, f_{b}^{*}$, such that, for any $n_{1}, \ldots, n_{p}, x_{1}, \ldots, x_{q}, z, f\left(n_{1}, \ldots, n_{p}, x_{1}, \ldots, x_{q}\right)=z$ if and only if there is some $m \in N$ such that $T\left(m, n_{1}, \ldots, n_{p}, x_{1}, \ldots, x_{q}\right)$ and $U\left(m, n_{1}, \ldots, n_{p}\right.$, $\left.x_{1}, \ldots, x_{q}\right)=x$. Furthermore, there is such a relation $T$ with the property that, for each $n_{1}, \ldots, n_{p}, x_{1}, \ldots, x_{q}$ there is at most one $m$ such that $T\left(m, n_{1}, \ldots, n_{p}, x_{1}, \ldots, x_{q}\right)$. Let $\left(F_{T}, G_{T}\right)$ and $\left(F_{U}, G_{U}\right)$ determine the representing function of $T$ and the function $U$ respectively as in Lemma 1. Now $T\left(m, n_{1}, \ldots, n_{p}, x_{1}, \ldots, x_{q}\right)$ if and only if, for some $k_{1} \in N$, $F_{T}\left(k_{1}\right)\left[m, n_{1}, \cdots, n_{p}, x_{1}, \cdots, x_{q}\right]$ and $G_{T}\left(k_{1}\right)\left[m, n_{1}, \cdots, n_{p}, x_{1}, \cdots, x_{q}\right]=0$. 
Also $U\left(m, n_{1}, \ldots, n_{p}, x_{1}, \ldots, x_{q}\right)=z$ if and only if, for some $k_{2} \in N$, $F_{U}\left(k_{2}\right)\left[m, n_{1}, \ldots, n_{p}, x_{1}, \ldots, x_{q}\right]$ and $G_{U}\left(k_{2}\right)\left[m, n_{1}, \ldots, n_{p}, x_{1}, \ldots, x_{q}\right]=z$. Let $Z$ be the formula $A^{0}\left(V_{1}\right) \wedge A^{0}\left(\pi V_{1}\right)$. If $z \in A^{*}$ then $Z[z]$ if and only if $z=0$. Now define recursive functions $F_{0}$ and $G_{0}$ on $N^{(p+3)}$ by:

$$
\begin{aligned}
F_{0}\left(m, k_{1}, k_{2}, n_{1}, \cdots, n_{p}\right)= & {\left[F_{T}\left(k_{1}\right) \wedge Z\left|G_{T}\left(k_{1}\right)\right| \wedge F_{U}\left(k_{2}\right)\right] } \\
& \cdot\left|\mathbf{m}, \mathbf{n}_{1}, \cdots, \mathbf{n}_{p}, \mathbf{v}_{1}, \cdots, \mathbf{V}_{q}\right|, \\
G_{0}\left(m, k_{1}, k_{2}, n_{1}, \cdots, n_{p}\right)= & G_{U}\left(k_{2}\right)\left|\mathbf{m}, \mathbf{n}_{1}, \cdots, \mathbf{n}_{p}, \mathbf{v}_{1}, \cdots, \mathbf{V}_{q}\right| \cdot
\end{aligned}
$$

Now $f\left(n_{1}, \ldots, n_{p}, x_{1}, \ldots, x_{q}\right)=z$ if and only if there are natural numbers $m, k_{1}$ and $k_{2}$ such that $F_{0}\left(m, k_{1}, k_{2}, n_{1}, \ldots, n_{p}\right)\left[x_{1}, \ldots, x_{q}\right]$ and $G_{0}\left(m, k_{1}, k_{2}, n_{1}, \ldots, n_{p}\right)\left[x_{1}, \ldots, x_{q}\right]=z$. Let $F\left(k, n_{1}, \ldots, n_{p}\right)=$ $\eta\left(F_{0}\left((k)_{0},(k)_{1},(k)_{2}, n_{1}, \ldots, n_{p}\right)\right)$. If $\eta\left(G_{0}\left((k)_{0},(k)_{1},(k)_{2}, n_{1}, \ldots, n_{p}\right)\right)$ is a term of $Q F$, let $G\left(k, n_{1}, \ldots, n_{p}\right)=\eta\left(G_{0}\left((k)_{0},(k)_{1},(k)_{2}, n_{1}, \ldots, n_{p}\right)\right)$. Otherwise, let $G\left(k, n_{1}, \ldots, n_{p}\right)=\mathrm{V}_{1}$. Let $\theta$ be some total recursive function such that, for any $m \in N, \theta(m)=m$ and let

$$
H\left(k, n_{1}, \cdots, n_{p}\right)=\theta\left(\eta\left(G_{0}\left((k)_{0},(k)_{1},(k)_{2}, n_{1}, \cdots, n_{p}\right)\right)\right) .
$$

Notice that $F, G$ and $H$ are recursive and that, for all $k_{,} n_{1}, \cdots, n_{p}$, $F\left(k, n_{1}, \cdots, n_{p}\right)$ and $G\left(k, n_{1}, \cdots, n_{p}\right)$ are respectively a formula and a term of $Q F$. Let $\left(n_{1}, \ldots, n_{p}, x_{1}, \ldots, x_{q}\right) \in N^{(p)} \times A^{(q)}$. If $f\left(n_{1}, \ldots, n_{p}, x_{1}, \ldots, x_{q}\right)=z$ then there are unique $m, k_{1}$ and $k_{2}$ such that $F_{0}\left(m_{0} k_{1}, k_{2}, n_{1}, \ldots, n_{p}\right)\left[x_{1}, \ldots, x_{q}\right]$ and, for those $m, k_{1}$ and $k_{2}, G_{0}\left(m, k_{1}, k_{2}, n_{1}, \ldots, n_{p}\right)\left[x_{1}, \ldots, x_{q}\right]:=z$. By

Lemma 2, using the fact that each $x_{i}$ is an element of $A, \eta\left(F_{0}\left(m_{0} k_{1}, k_{2}, n_{1}, \cdots, n_{p}\right)\right)$ - $\left[x_{1}, \cdots, x_{q}\right]$. In fact, letting $k=\left\langle m, k_{1}, k_{2}\right\rangle, k=\mu k F\left(k, n_{1}, \cdots, n_{p}\right)\left[x_{1}, \cdots, x_{q}\right]$. If $f$ is into $A$ then $z \in A$ so, by Lemma $2, \eta\left(G_{0}\left(m, k_{1}, k_{2}, n_{1}, \ldots, n_{p}\right)\right)$ is a term of $Q F$ and $\eta\left(G_{0}\left(m, k_{1}, k_{2}, n_{1}, \ldots, n_{p}\right)\right)\left[x_{1}, \ldots, x_{q}\right]=z$, i.e., $G\left(k, n_{1}, \ldots, n_{p}\right)\left[x_{1}, \ldots, x_{q}\right]=z$. To summarize, if $\left(n_{1}, \ldots, n_{p}, x_{1}, \ldots, x_{q}, z\right)$ $\epsilon N^{(p)} \times A^{(q+p)}$ and if $f\left(n_{1}, \ldots, n_{p}, x_{1}, \ldots, x_{q}\right)=z$ then

$$
G\left(\mu k F\left(k, n_{1}, \cdots, n_{p}\right)\left[x_{1}, \cdots, x_{q}\right], n_{1}, \cdots, n_{p}\right)\left[x_{1}, \cdots, x_{q}\right]=z .
$$

Now suppose that $f$ is into $N$. Then $z \in N$ so, by Lemma 2,

$$
\eta\left(G_{0}\left(m, k_{1}, k_{2}, n_{1}, \cdots, n_{p}\right)\right)=\mathbf{z} \text {. }
$$

Therefore, $H\left(k, n_{1}, \cdots n_{p}\right)=z$. To summarize, if $\left(z, n_{1}, \cdots, n_{p}, x_{1}, \cdots, x_{q}\right)$ $\epsilon N^{(p+1)} \times A^{(q)}$ and $f\left(n_{1}, \cdots, n_{p}, x_{1}, \cdots, x_{q}\right)=z$ then

$$
H\left(\mu k F\left(k, n_{1}, \cdots, n_{p}\right)\left[x_{1}, \cdots, x_{q}\right], n_{1}, \cdots, n_{p}\right)=z .
$$

Now if $f$ is into $A$ and $G\left(\mu k F\left(k, n_{1}, \ldots, n_{p}\right)\left[x_{1}, \ldots, x_{q}\right], n_{1}, \ldots, n_{p}\right)\left[x_{1}, \ldots, x_{q}\right]=z$, 
then there is some $k$ such that $F\left(k, n_{1}, \ldots, n_{p}\right)\left[x_{1}, \ldots, x_{q}\right]$. It follows that, letting $m=(k), T\left(m, n_{1}, \ldots, n_{p}, x_{1}, \ldots, x_{q}\right)$ and hence that $f\left(n_{1}, \ldots, n_{p}\right.$, $\left.x_{1}, \ldots, x_{q}\right)$ is defined and equal so some $z_{1}$. Put, as has just been shown, it follows that

$$
G\left(\mu k F\left(k, n_{1}, \ldots: n_{p}\right)\left[x_{1}, \cdots, x_{q}\right], n_{1}, \cdots, n_{p}\right)\left[x_{1}, \cdots, x_{q}\right]=z_{1} .
$$

- Hence $z=z_{1}$. This concludes the proof of part (a) of the theorem. Similarly, if $f$ is into $A$ and $H\left(\mu k F\left(k, n_{1}, \cdots, n_{p}\right)\left[x_{1}, \cdots, x_{q}\right], n_{1}, \cdots, n_{p}\right)=z$ then $f\left(n_{1}, \cdots, n_{p}, x_{1}, \cdots, x_{q}\right)$ is defined and must equal $z$. This concludes the proof of part (b) of the theorem.

The converse to Theorem 1 will be proved in $\$ 7$. It follows from the prime computability of (i) all recursive functions and (ii) the satisfaction relation for formulas of $Q F$.

6. Search computable functions. Given a term $t$ of $Q F^{*}$ with variables from $\mathbf{V}_{q+1}, \ldots, \mathbf{v}_{q+k}$ and given elements $x_{1}, \ldots, x_{k}$ of $A^{*}$, let $t\left[x_{1}, \ldots, x_{k}\right]_{q}$ be that element of $A^{*}$ which the term $t$ represents when $V_{q+1}, \ldots, V_{q+k}$ are interpreted as $x_{1}, \ldots, x_{k}$ respectively.

Lemma 3. There are total recursive functions $\alpha$ and $\beta$ such that, for natural numbers $q$ and $n, \alpha(q, n)$ is a term of $T m^{*}$ with variables from $\mathbf{V}_{q+1}, \ldots, \mathbf{V}_{q+\beta(n)}$ (or $\alpha(q, n)=0$ if $\left.\beta(n)=0\right)$ and sucb that, given any $w \in A^{*}$, there is some $n \in N$ and some $x_{1}, \ldots, x_{\beta(n)} \in A$ such that, for any $q \in N$, $\alpha(q, n)\left[x_{1}, \ldots, x_{\beta(n)}\right]_{q}=w$.

Proof. Define $\alpha$ and $\beta$ as follows:

$$
\begin{aligned}
& \alpha(q, n)=\left\{\begin{array}{l}
\mathbf{V}_{q+k+1} \text { if } n=k, \\
\mathbf{p}(\alpha(q, u), \alpha(q+\beta(u), v)) \text { if } n=\langle u, v\rangle, \\
0 \quad \text { if } n \text { is not a sequence number of length } 1 \text { or } 2 .
\end{array}\right. \\
& \beta(n)= \begin{cases}k+1 & \text { if } n=\langle k\rangle, \\
\beta(u)+\beta(v) & \text { if } n=\langle u, v\rangle, \\
.0 & \text { if } n \text { is not a sequence number of length } 1 \text { or } 2 .\end{cases}
\end{aligned}
$$

The functions $\alpha$ and $\beta$ are clearly recursive, in fact, both $\alpha$ and $\beta$ are primitive recursive. By induction on $n$, it is immediate that $\alpha(q, n)$ is a term of $T m^{*}$ with variables from $\mathbf{V}_{q+1}, \ldots, \mathrm{V}_{q+\beta(n)}$. We show by $A_{\text {-induction that, for }}^{*}$ 
$w \in A^{*}$; for any $q \in N$ there is some $n \in N$ and some $x_{1}, \ldots, x_{\beta(n)} \in A$ such that $\alpha(q, n)\left[x_{1}, \ldots, x_{\beta(n)}\right]_{q}=w$. If $w \in A$, let $n=\langle 0\rangle$ so $\alpha(q, n)=\mathbf{V}_{q+1}$, $\beta(n)=1$ and $\alpha(q, n)[w]_{q}=w$. If $w=0$, let $n=0$ so $\alpha(q, n)=0$ and $\beta(n)=0$. Suppose $w=(s, t)$ and the induction hypothesis holds for $s$ and $t$. Let $q$ be fixed. Choose $u, x_{1}, \ldots, x_{\beta(u)}$ such that $\alpha(q, u)\left[x_{1}, \ldots, x_{\beta(u)}\right]_{q}=s$ and choose $v, y_{1}, \ldots, y_{\beta(v)}$ such that $\alpha(q+\beta(u), v)\left[y_{1}, \ldots, y_{\beta(v)}\right]_{q+\beta(u)}=y$. Let $n=\langle u, v\rangle$. Now

$$
a(q, n)\left[x_{1}, \cdots, x_{\beta(u)} y_{1}, \cdots, y_{\beta(v)}\right]_{q}=\left(s^{\prime}, t^{\prime}\right)
$$

where

$$
s^{\prime}=\alpha(q, u)\left[x_{1}, \cdots, x_{\beta(u)}, y_{1}, \cdots, y_{\beta(v)}\right]_{q}
$$

and

$$
t^{\prime}=\alpha(q+\beta(u), v)\left[x_{1}, \cdots, x_{\beta(u)}, y_{1}, \cdots, y_{\beta(v)}\right]_{q^{\bullet}}
$$

But the variables of $\alpha(q, u)$ are from $\mathbf{V}_{q+1}, \ldots, \mathbf{V}_{q+\beta(u)}$ and the variables of $\alpha(q+\beta(u), v)$ are from $\mathbf{V}_{q+\beta(u)+1}, \ldots, \mathbf{V}_{q+\beta(u)+\beta(v)}$ so $s=\alpha(q, u)\left[x_{1}, \ldots, x_{\beta(u)}\right]_{q}=s$ and $t^{\prime}=\alpha(q+\beta(u), v)\left[y_{1}, \cdots, y_{\beta(v)}\right]_{q+\beta(u)}^{q}=t$. Therefore $\left(s^{\prime}, t^{\prime}\right)=w$. This concludes the proof of Lemma 3.

Theorem 2. There is a recursive function $\gamma$ sucb that: (a) if $f$ is an U-search-computable function from $N^{(p)} \times A^{(q)}$ into $A$, then there are total recursive $p+1$ place functions $F$ and $G$ sucb that, for any $\left(k, n_{1}, \cdots, n_{p}\right) \epsilon$ $N^{(p+1)}, F\left(k, n_{1}, \ldots, n_{p}\right)$ and $G\left(k, n_{1}, \ldots, n_{p}\right)$ are respectively $a$ formula and a term of $Q F$ with variables from $\mathbf{V}_{1}, \ldots, \mathbf{V}_{g+\gamma(k)}$ and such that, for any $\left(n_{1}, \ldots, n_{p}, x_{1}, \cdots, x_{q}, z\right) \in N^{(p)}+A^{(q+1)^{q+\gamma(k)}}, f\left(n_{1}, \ldots, n_{p}, x_{1}, \ldots, x_{q}\right)=z$ if and only if there is some natural number $k$ and some sequence $y_{1}, \ldots, y_{\gamma(k)}$ of elements of $A$ such that

$$
F\left(k, n_{1}, \cdots, n_{p}\right)\left[x_{1}, \cdots, x_{q}, y_{1}, \cdots, y_{\gamma(k)}\right]
$$

and

$$
G\left(k, n_{1}, \cdots, n_{p}\right)\left[x_{1}, \cdots, x_{q}, y_{1}, \cdots, y_{\gamma(k)}\right]=z .
$$

(b) if $f$ is an Usearch-computable function from $N^{(p)} \times A^{(q)}$ into $N$ then there are total recursive functions $F$ and $H$ such that, for any $\left(k, n_{1}, \ldots, n_{p}\right) \epsilon$ $N^{(p+1)}, F\left(k, n_{1}, \ldots, n_{p}\right)$ is a formula of $Q F$ with variables from $\mathbf{V}_{1}, \ldots$, $\mathbf{V}_{q+\gamma(k)}$ and such that, for any $\left(z, n_{1}, \cdots, n_{p}, x_{1}, \cdots, x_{q}\right) \in N^{(p+1)} \times A^{(q)}$, $f\left(n_{1}, \ldots, n_{p}, x_{1}, \ldots, x_{q}\right)=z$ if and only if there is some natural number $k$ and some sequence $y_{1}, \ldots, y_{\gamma(k)}$ of elements of $A$ such that $F\left(k, n_{1}, \ldots, n_{p}\right)$. $\left[x_{1}, \ldots, x_{q}, y_{1}, \ldots, y_{\gamma(k)}\right]$ and $H\left(k, n_{1}, \ldots, n_{p}\right)=z$. 
Proof. Let $\eta$ be as in Lemma 2. Let $\alpha$ and $\beta$ be as in Lemma 3. Let $\theta$ be a total recursive function such that, for each $z \in N, \theta(\mathrm{z})=z$. Let $\gamma(k)=$ $\beta\left((k)_{0}\right)$. If $f$ is any 2 -search-computable function into $A$ or into $N$, then, by the normal form theorem for search computable functions, there is a relation $T$ and a function $U$, each absolutely primitive computable in $g_{1}^{*}, \ldots, g_{a}^{*}, f_{1}^{*}, \ldots, f_{b}^{*}$, such that, for all $n_{1}, \ldots, n_{p}, x_{1}, \ldots, x_{q}, z, f\left(n_{1}, \ldots, n_{p}, x_{1}, \ldots, x_{q}\right)=z$ if and only if there is some $w \in A^{*}$ such that $T\left(n_{1}, \ldots, n_{p}, x_{1}, \ldots, x_{q}, w\right)$ and $U\left(n_{1}, \ldots, n_{p}, x_{1}, \ldots, x_{q}, w\right)=z$. Let $\left(F_{T}, G_{T}\right)$ and $\left(F_{U}, G_{U}\right)$ determine the representing function of $T$ and the function $U$ respectively as in Lemma 1. Define functions $F_{0}$ and $G_{0}$ on $N^{(p+3)}$ by:

$$
\begin{aligned}
F_{0}\left(m, k_{1}, k_{2}, n_{1}, \cdots, n_{p}\right)= & \left(F_{T}\left(k_{1}\right) \wedge Z\left|G_{T}\left(k_{1}\right)\right| \wedge F_{U}\left(k_{2}\right)\right) \\
& \cdot\left|\mathbf{n}_{1}, \cdots, \mathbf{n}_{p}, \mathbf{V}_{1}, \cdots, \mathbf{V}_{q}, \alpha(q, m)\right|, \\
G_{0}\left(m, k_{1}, k_{2}, n_{1}, \cdots, n_{p}\right)= & G_{U}\left(k_{2}\right)\left|\mathbf{n}_{1}, \cdots, \mathbf{n}_{p}, \mathbf{V}_{1}, \cdots, \mathbf{V}_{q}, \alpha(q, m)\right| .
\end{aligned}
$$

Now if

$$
F_{0}\left(m, k_{1}, k_{2}, n_{1}, \cdots, n_{p}\right)\left[x_{1}, \cdots, x_{q}, y_{1}, \cdots, y_{\beta(m)}\right]
$$

and

$$
G_{0}\left(m, k_{1}, k_{2}, n_{1}, \cdots, n_{p}\right)\left[x_{1}, \cdots, x_{q}, y_{1}, \cdots, y_{\beta(m)}\right]=z
$$

then, letting $w=\alpha(q, m)\left[y_{1}, \ldots, y_{\beta(m)}\right]_{q}$,

$$
\begin{aligned}
& F_{T}\left(k_{1}\right)\left[n_{1}, \cdots, n_{p}, x_{1}, \cdots, x_{q}, w\right], \quad G_{T}\left(k_{1}\right)\left[n_{1}, \cdots, n_{p}, x_{1}, \cdots, x_{q}, w\right]=0, \\
& F_{U}\left(k_{2}\right)\left[n_{1}, \cdots, n_{p}, x_{1}, \cdots, x_{q}, w\right], \quad G_{U}\left(k_{2}\right)\left[n_{1}, \cdots, n_{p}, x_{1}, \cdots, x_{q}, w\right]=z .
\end{aligned}
$$

Therefore, $T\left(n_{1}, \ldots, n_{p}, x_{1}, \ldots, x_{q}, w\right)$ and $U\left(n_{1}, \ldots, n_{p}, x_{1}, \ldots, x_{q}, w\right)=z$ so $f\left(n_{1}, \ldots, n_{p}, x_{1}, \ldots, x_{q}\right)=z$. On the other hand, if $f\left(n_{1}, \cdots, n_{p}, x_{1}, \ldots, x_{q}\right)=z$ then there is some $w \in A^{*}$ and some natural numbers $k_{1}$ and $k_{2}$ such that $F_{T}\left(k_{1}\right)\left[n_{1}, \ldots, n_{p}, x_{1}, \ldots, x_{q}, w\right], G_{T}\left(k_{1}\right)\left[n_{1}, \ldots, n_{p}, x_{1}, \ldots, x_{q}, w\right]=0$, $F_{U}\left(k_{2}\right)\left[n_{1}, \ldots, n_{p}, x_{1}, \ldots, x_{q}, w\right]$ and $G_{U}\left(k_{2}\right)\left[n_{1}, \ldots, n_{p}, x_{1}, \ldots, x_{q}, w\right]=z$. Pick some $m \in N$ and some sequence $y_{1}, \ldots, y_{\beta(m)}$ of members of $A$ such that $\alpha(q, m)\left[y_{1}, \cdots, y_{\beta(m)}\right]_{q}=w$, then

$$
F_{0}\left(m, k_{1}, k_{.2}, n_{1}, \cdots, n_{p}\right)\left[x_{1}, \cdots, x_{q}, y_{1}, \ldots, y_{\beta(m)}\right]
$$

and

$$
G_{0}\left(m, k_{1}, k_{2}, n_{1}, \cdots, n_{p}\right)\left[x_{1}, \cdots, x_{q}, y_{1}, \cdots, y_{\beta(m)}\right]=z \text {. }
$$


Now let

$$
F\left(k, n_{1}, \cdots, n_{p}\right)=\eta \eta\left(F_{0}\left((k)_{0},(k)_{1},(k)_{2}, n_{1}, \cdots, n_{p}\right)\right)
$$

and

$$
G\left(k, n_{1}, \cdots, n_{p}\right)=\eta\left(G_{0}\left((k)_{0},(k)_{1},(k)_{2}, n_{1}, \cdots, n_{p}\right)\right) .
$$

For each $\left(k, n_{1}, \ldots, n_{p}\right) \in N^{(p+1)}, F\left(k, n_{1}, \ldots, n_{p}\right)$ is a formula of $Q F$ with variables from $V_{1}, \ldots, V_{q+\gamma(k)}$. In case $f$ is a function into $A$ then, for each $\left(k, n_{1}, \ldots, n_{p}\right) \in N^{(p+1)}, G\left(k, n_{1}, \ldots, n_{p}\right)$ is a term of $Q F$ with variables from $\mathbf{V}_{1}, \ldots, \mathbf{V}_{q+\gamma(k)}$. Furthermore, for each $\left(n_{1}, \ldots, n_{p}, x_{1}, \ldots, x_{q}, z\right) \in N^{(p)} \times$ $A^{(q+1)}, f\left(n_{1}, \ldots, n_{p}, x_{1}, \ldots, x_{q}\right)=z$ if and only if there is some $k \in N$ and some sequence $y_{1}, \cdots, y_{\gamma(k)}$ of elements of $A$ such that

$$
F\left(k, n_{1}, \cdots, n_{p}\right)\left[x_{1}, \cdots, x_{q}, y_{1}, \cdots, y_{\gamma(k)}\right]
$$

and

$$
G\left(k, n_{1}, \cdots, n_{p}\right)\left[x_{1}, \cdots, x_{q}, y_{1}, \cdots, y_{\gamma(k)}\right]=z .
$$

In case $f$ is a function into $N$ then, for each $\left(k, n_{1}, \ldots, n_{p}\right) \in N^{(p+1)}$, $G\left(k, n_{1}, \ldots, n_{p}\right)$ is a numeral of $T m^{*}$. Furthermore, for each $\left(z, n_{1}, \ldots, n_{p}\right.$, $\left.x_{1}, \ldots, x_{q}\right) \in N^{(p+1)} \times A^{(q)}, f\left(n_{1}, \ldots, n_{p}, x_{1}, \ldots, x_{q}\right)=z$ if and only if there is some $k \in N$ and some sequence $y_{1}, \ldots, y_{\gamma(k)}$ of elements of $A$ such that $F\left(k, n_{1}, \ldots, n_{p}\right)\left[x_{1}, \ldots, x_{q}, y_{1}, \ldots, y_{\gamma(k)}\right]$ and $G\left(k, n_{1}, \ldots, n_{p}\right)=z$. Letting $H\left(k, n_{1}, \ldots, n_{p}\right)=\theta\left(G\left(k, n_{1}, \ldots, n_{p}\right)\right)$ and leaving the reader to verify that $F, G$ and $H$ are recursive, the theorem is proved.

Define the language $E x$ as follows:

(1) The terms of $E_{x}$ are just the terms of $Q F$.

(2) The formulas of $E_{x}$ are defined inductively by:

(i) Every formula of $Q F$ is a formula of $E x$.

(ii) If $\phi$ is a forumla of $E x$ and $x$ is a variable then $\langle 11, x, \phi\rangle$ is a formula of $E_{x}$ (denoted by $\exists x \phi$ and having the corresponding interpretation).

Corollary to Theorem 2. If $f$ is an $\mathrm{U}$-searcb-computable function from $N^{(p)} \times A^{(q)}$ into $N$ then there are total recursive functions $F$ and $H$ such that, for any $\left(k, n_{1}, \ldots, n_{p}\right) \in N^{(p+1)}, F\left(k, n_{1}, \ldots, n_{p}\right)$ is a formula of $E x$ and, for any $\left(n_{1}, \ldots, n_{p}, x_{1}, \ldots, x_{q}\right) \in N^{(p)} \times A^{(q)}, f\left(n_{1}, \ldots, n_{p}, x_{1}, \ldots, x_{q}\right)=$ $H\left(\mu k F\left(k, n_{1}, \ldots, n_{p}\right)\left[x_{1}, \ldots, x_{q}\right], n_{1}, \ldots, n_{p}\right)$.

7. The converses to Theorems 1 and 2. Putting aside our use of " $\left\langle x_{0}, \ldots, x_{k-1}\right\rangle "$ to denote $\Pi_{i<k} P_{i}^{x_{i}}$, we now let " $\left(x_{0}, \ldots, x_{k-1}\right)$ " denote that element of $A^{*}$ which codes " $\left(x_{0}, \ldots, x_{k-1}\right)$ " as in [3]. If $x=\left\langle x_{0}, \ldots, x_{k-1}\right\rangle$, 
let $\operatorname{lh}(x)=k$ and $(x)_{i}=x_{i}(i=0, \ldots, k-1)$. Let Val be the partial function on $A^{*}$ defined by

$$
\operatorname{Val}(t, x)=\left\{\begin{aligned}
t\left[x_{1}, \cdots, x_{q}\right] & \text { if } t \text { is a term of } Q F \text { and } x=\left\langle x_{1}, \cdots, x_{q}\right\rangle, \\
& \text { where each } x_{i} \text { is an element of } A, \\
\text { undefined otherwise. } &
\end{aligned}\right.
$$

Let Sat be the relation on $A^{*}$ defined by: Sat $(\phi, x)$ if and only if $\phi$ is a formula of $Q F, x$ is of the form $\left\langle x_{1}, \ldots, x_{q}\right\rangle$, with each $x_{i} \in A$, and $\phi\left[x_{1}, \ldots, x_{q}\right]$. It is a consequence of the recursion theorem for prime computable functions that $\mathrm{Val}$ and Sat are absolutely prime computable (cf. [3]). Every recursive function is prime computable (cf. [3]), and the set of prime computable functions is closed under the minimalization operator. This is sufficient to give us

Theorem 3. (a) If $F$ and $G$ are $p+1$ place total recursive functions and if, for any $\left(k, n_{1}, \ldots, n_{p}\right) \in N^{(p+1)}, F\left(k, n_{1}, \ldots, n_{p}\right)$ and $G\left(k, n_{1}, \ldots, n_{p}\right)$ are respectively a formula and a term of $Q F$ with variables from $\mathbf{v}_{1}, \ldots, \mathbf{v}_{q}$, then the function $f$ from $N^{(p)} \times A^{(q)}$ into $A$ defined by

$$
\begin{aligned}
& f\left(n_{1}, \cdots, n_{p}, x_{1}, \cdots, x_{q}\right) \\
& \quad=G\left(\mu k F\left(k, n_{1}, \cdots, n_{p}\right)\left[x_{1}, \cdots, x_{q}\right], n_{1}, \cdots, n_{p}\right)\left[x_{1}, \cdots, x_{q}\right]
\end{aligned}
$$

is U-prime-computable.

(b) If $F$ and. $H$ are $p+1$ place total recursive functions and, for every $\left(k, n_{1}, \ldots, n_{p}\right) \in N^{(p+1)}, F\left(k, n_{1}, \ldots, n_{p}\right)$ is a formula of $Q F$ with variables from $\mathbf{V}_{1}, \ldots, \mathbf{V}_{q}$, then the function $f$ from $N^{(p)} \times A^{(q)}$ into $N$ defined by $f\left(n_{1}, \ldots, n_{p}, x_{1}, \ldots, x_{q}\right)=H\left(\mu k F\left(k, n_{1}, \ldots, n_{p}\right)\left[x_{1}, \ldots, x_{q}\right], n_{1}, \ldots, n_{p}\right)$ is U-prime-computable.

Theorem 4. (a) If $F$ and $G$ are $p+1$ place total recursive functions and $\gamma$ is a total recursive function and if, for any $\left(k, n_{1}, \ldots, n_{p}\right) \in N^{(p+1)}$, $F\left(k, n_{1}, \ldots, n_{p}\right)$ and $G\left(k, n_{1}, \ldots, n_{p}\right)$ are respectively a formula and a term of QF with variables from $\mathbf{V}_{1}, \ldots, \mathbf{V}_{q+\gamma(k)}$, then the (partial, multiple valued) function $f$ from $N^{(p)} \times A^{(q)}$ into $A$ defined by:

$$
\begin{aligned}
& f\left(n_{1}, \cdots, n_{p}, x_{1}, \cdots, x_{q}\right)=z \text { if and only if, for some } k \in N \text { and some } \\
& y_{1}, \cdots, y_{\gamma(k)} \in A, F\left(k, n_{1}, \cdots, n_{p}\right)\left[x_{1}, \cdots, x_{q}, y_{1}, \cdots, y_{\gamma(k)}\right] \text { and } \\
& G\left(k, n_{1}, \cdots, n_{p}\right)\left[x_{1}, \cdots, x_{q}, y_{1}, \cdots, y_{\gamma(k)}\right]=z
\end{aligned}
$$

is $\mathcal{Q}$-search-computable. (b) If $F$ and $H$ are $p+1$ place total recursive functions, 
and $\gamma$ is a total recursive function, and if, for any $\left(k, n_{1}, \ldots, n_{p}\right) \in N^{(p+1)}$, $F\left(k, n_{1}, \ldots, n_{p}\right)$ is a formula of $Q F$ with variables from $\mathbf{V}_{1}, \ldots, \mathbf{V}_{q+\gamma(k)}$, then the function $f$ from $N^{(p)} \times A^{(q)}$ into $N$ defined by:

$f\left(n_{1}, \cdots, n_{p}, x_{1}, \cdots, x_{q}\right)=z$ if and only if there is some $k \in N$ and some $y_{1}, \cdots, y_{k} \in A$ such that $F\left(k, n_{1}, \cdots, n_{p}\right)\left[x_{1}, \cdots, x_{q}, y_{1}, \cdots, y_{\gamma(k)}\right]$ and $H\left(k, n_{1}, \cdots, n_{p}\right)=z$

is Ir-search-computable.

Proof. Let $\nu$ be the search operator of [3]. Let $f$ be defined from $F$ and $G$ as in part (a). Define $y=y\left(n_{1}, \ldots, n_{p}, x_{1}, \ldots, x_{q}\right)=\nu w$ (sequence $(w) \&$ $(w)_{0} \in N \&(\forall i<\operatorname{lh}(w)-1)\left((w)_{i+1} \in A \& \operatorname{lh}(w)=y\left((w)_{0}\right)+1 \times F(w)_{0}, n_{1}, \cdots, n_{p}\right)$ $\left.\cdot\left[x_{1}, \ldots, x_{q},(w)_{1}, \ldots,(w)_{\gamma\left((w)_{0}\right)}\right]\right)$. Now $y$ is $\mathcal{U}$-search-computable and

$$
\begin{aligned}
& f\left(n_{1}, \cdots, n_{p}, x_{1}, \cdots, x_{q}\right) \\
& \quad=G\left((y)_{0}, n_{1}, \cdots, n_{p}\right)\left[x_{1}, \cdots, x_{q},(y)_{1}, \cdots,(y)_{\gamma\left((y)_{0}\right)}\right] .
\end{aligned}
$$

Hence $f$ is 2 -search-computable. Now suppose $f$ is defined from $F$ and $H$ as in part (b). Let $y$ be as above. Then $f\left(n_{1}, \ldots, n_{p}, x_{1}, \ldots, x_{q}\right)=$ $H\left((y)_{0}, n_{1}, \ldots, n_{p}\right)$ so $f$ is $\mathcal{U}$-search-computable.

8. Computability and the constructible $L_{\omega_{1}, \omega}$ The infinitary language "constructible $L_{\omega_{1}, \omega}$ " (cf. [4]) has finitary quantification and infinitary disjunctions W $\Omega$ of nonempty, recursively enumerable sets $\Omega$ of formulas. We consider certain sublanguages of constructible $L_{\omega_{1}, \omega^{\circ}}$ An existential formula is a formula of the language $E_{x}$ of $\$ 6$. An $W$-formula is a formula of the form $W \Omega$, where $\Omega$ is a recursively enumerable set of formulas of $Q F$ all of the variables of which lie in some finite set. An W/ J-formula is a formula of the form $W \Omega$, where $\Omega$ is a recursively enumerable set of existential formulas all of the free variables of which lie in some finite set.

Recall that a relation is called semi-20-prime-computable (semi-20-search-

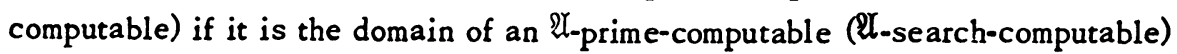
function. By Theorems 1 and 3 (2 and 4), a $q$ place relation $R$ on $A$ is semiUl-prime-computable (semi-थ-search-compuable) if and only if there is a total recursive function $F$ into the formulas of $Q F(E x)$ such that, for any $\left(x_{1}, \ldots, x_{q}\right) \in A^{(q)}, R\left(x_{1}, \ldots, x_{q}\right)$ if and only if, for some $k, F(k)\left[x_{1}, \ldots, x_{q}\right]$. An immediate consequence is the next theorem.

Theorem 5. (a) A relation on $A$ is definable in $\mathfrak{A}$ by an W-formula if and only 
if it is semi-2r-prime-computable. (b) A relation on $A$ is definable in $\mathcal{O}$ by an WJ-formula if and only if it is semi-2t-search-computable. (1)

Remark. There are many "pathological" cases that might be considered. For example, if $\mathcal{Q}$ has no "given" relations, then, writing $\mathrm{n}$ for $n_{1}, \ldots, n_{p}$ and $\mathbf{x}$ for $x_{1}, \ldots, x_{q}$, (a) the थ-prime-computable functions $f: N^{(p)} \times A^{(q)} \rightarrow N$ are those of the form $f(n, x)=g(n)$, for some partial recursive $g$, and (b) the 2l-prime-computable functions $f: N^{(p)} \times A^{(q)} \rightarrow A$ are those of the form $f(\mathbf{n}, \mathbf{x})=g(\mathbf{n})[\mathbf{x}]$, for some partial recursive function $g$ into the terms of $Q F$. Hence an $थ$-prime-computable function $f: A^{(q)} \rightarrow N$ is nowhere defined or constant and an 2 -prime-computable function $f: A^{(q)} \rightarrow A$ is nowhere defined or is a composition of "given" functions. If, on the other hand, थ has no "given"

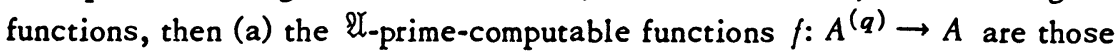
which can be defined by cases:

$$
f(\mathrm{x})= \begin{cases}x_{1} & \text { if } \phi_{1}[\mathrm{x}], \\ \dot{0} & \\ x_{q} & \text { if } \phi_{q}[\mathrm{x}]\end{cases}
$$

where $\phi_{1}, \ldots, \phi_{q}$ are formulas of $Q F$ and if $i<j$ then " $\left.\phi_{j} \Rightarrow\right\urcorner \phi_{i}$ " is valid and (b) the U-prime-computable relations $R \subseteq A^{(q)}$ are those definable by formulas of $Q F$. Other such special cases are left for the amusement of the reader.

\section{REFERENCES}

1. D. Lacombe, Recursion theoretic structure for relational systems, Logic Colloquium '69 (Proc. Summer School and Colloq., Manchester, 1969), North-Holland Amsterdam, 1971, pp. 3-17. MR 43 \# 1823.

2. Y. N. Moschovakis, Abstract computability and invariant definability, J. Symbolic Logic 34 (1969), 605-633. MR 42 \#5791.

3. - Abstract first order computability. I, Trans. Amer. Math. Soc. 138 (1969), 427-464. MR 39 \#5362.

4. G. Takeuti and A. Kino, On predicates with constructive infinitely long expressions, J. Math. Soc. Japan 15 (1963), 176-190. MR $27 \# 5676$.

DEPARTMENT OF MATHEMATICS, CALIFORNIA STATE UNIVERSITY, LOS ANGELES, CALIFORNIA 90032

( 1 ) For the case that $\mathfrak{A}$ is a relational structure with equality, Theorem $5(b)$ is closely related to results of $D_{\text {aniel }} L_{\text {acombe }}$ and Yiannis Moschovakis. Lacombe asserts in [1] that a relation is "recursive in $R_{1}, \ldots, R_{a}$ " in a sense defined by Fräisse, if and only if both it and its complement are W/ definable from $R_{1}, \ldots, R_{e^{\prime}}=$. Moschovakis shows in [2] that a relation is Fraisse recursive in $R_{1}, \ldots, R_{a}$ if and only if it is search computable in $R_{1}, \ldots, R_{a},=$. 